\title{
Isolated peripunctal sebaceous gland carcinoma in a young adult: An unusual presentation
}

\author{
Tarjani V. Dave ${ }^{1,2}$, Adit Gupta1,2, Swathi Kaliki ${ }^{1,2}$, Dilip Mishra ${ }^{3}$
}

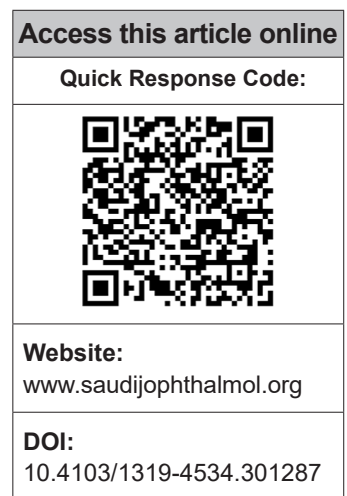

${ }^{1}$ Operation Eyesight, Universal Institute of Eye Cancer, L V Prasad Eye Institute, ${ }^{20}$ phthalmic Plastic Surgery Service, L V Prasad Eye Institute, ${ }^{3}$ Ophthalmic Pathology Laboratory, L V Prasad Eye Institute, Kallam Anji Reddy Campus, Road No.

2, Banjara Hills, Hyderabad 500 034, Telangana, India

Address for correspondence: Tarjani V. Dave,

L V Prasad Eye Institute, Kallam Anji Reddy Campus, Road No. 2, Banjara Hills, Hyderabad - 500034 , Telangana, India

E-mail: tvdeye@gmail.com

Submitted: 29-May-2018

Revised: 20-Aug-2018

Accepted: 15-May-2019

Published: 22-Nov-2020

\begin{abstract}
:
Sebaceous gland carcinoma (SGC) of the eyelid is one of the leading peri-ocular eyelid malignancies in AsianIndians. It usually affects elderly women and has a high rate of local recurrence, regional and distant metastasis. This is partly attributable to a delay in clinical diagnosis since it mimics more benign conditions of the eyelid like chalazia. To the best of our knowledge, this is the first case report of a peri-punctal SGC. Atypical location and the young age of the patient resulted in a relatively conservative excisional biopsy under frozen section control and eyelid reconstruction with a favorable outcome.
\end{abstract}

Keywords:

Sebaceous gland carcinoma, eyelid malignancies, clinical diagnosis, India

Cebaceous gland carcinoma (SGC) is a $\checkmark$ malignant neoplasm that originates from glandular epithelium of the sebaceous glands. It is a relatively common tumor of the eyelid in the Southeast Asian region as compared to the West. ${ }^{[1]}$ The metastasis-related mortality described in sebaceous cell carcinoma varies from $2 \%$ to $41 \% .^{[2,3]}$ Periocular SGC can originate from the meibomian glands, glands of Zeis, caruncle, pilosebaceous unit of facial skin and pilosebaceous unit of eyebrow hair. ${ }^{[2]}$ Although, upper eyelid is the commonest site for SGC to arise ${ }^{[4]}$ from, to the best of our knowledge peri-punctal location of this malignancy has not been reported previously. We report a case of histopathologically confirmed peri-punctal SGC in a young lady.

A 27-year-old lady presented to us with a recurrent mass lesion in the left upper eyelid present since a year [Figure 1a]. She gave a prior history of incision and curettage done elsewhere for the same condition twice. After both procedures, she noticed a recurrence of the lesion within 3 months. The mass was peri-punctal in location and involved the tarsal conjunctiva. The

This is an open access journal, and articles are distributed under the terms of the Creative Commons Attribution-NonCommercial-ShareAlike 4.0 License, which allows others to remix, tweak, and build upon the work non-commercially, as long as appropriate credit is given and the new creations are licensed under the identical terms.

For reprints contact:WKHLRPMedknow_reprints@wolterskluwer.com overlying skin showed loss of cilia at the eyelid margin and rounding of the posterior eyelid margin [Figure 1b]. Rest of the eyelid margin was normal. There was no diffuse conjunctival component associated with the nodule. Visual acuity, Hertels exophthalmometry measurements and extraocular muscle motility were normal. There was no regional lymphadenopathy and systemic workup revealed no metastasis. The lacrimal system saline irrigation was patent through the lower punctum and the saline irrigation through upper punctum was not checked for in view of the peri-punctal nature of the lesion. There was no history of bloody epiphora and there was no regurgitation on pressure over the lacrimal sac. Incision biopsy of the peri-punctal tissue was planned under frozen section control, which confirmed the diagnosis of SGC. The tumor was removed en-bloc with $4 \mathrm{~mm}$ margins again with frozen section control to rule out involvement of the margins. The upper punctum and canaliculus were sacrificed in securing margin control. Double freeze thaw cryotherapy was applied to all the skin and conjunctival margins as well as the canalicular cut end. Eyelid reconstruction was performed in the same sitting, wherein the posterior lamella was reconstructed using a sliding tarsoconjunctival flap and the anterior lamella was reconstructed

How to cite this article: DaveTV, Gupta A, Kaliki S, Mishra D. Isolated peripunctal sebaceous gland carcinoma in a young adult; an unusual presentation. Saudi J Ophthalmol 2020;34:73-5. 


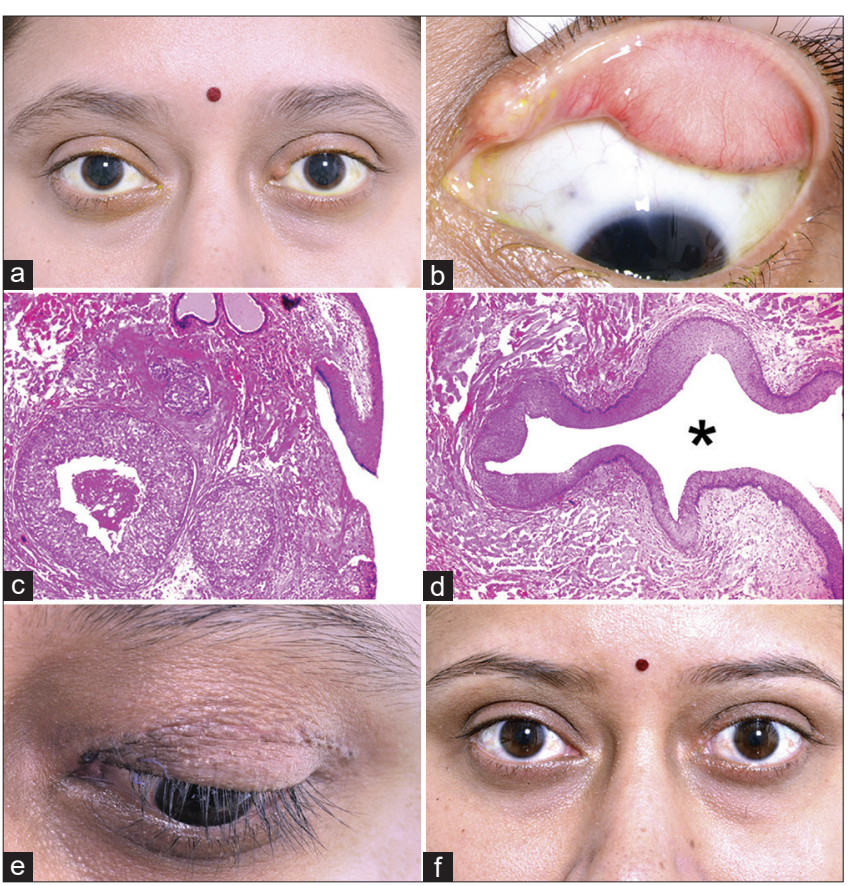

Figure 1: Per-operative, histopathology and post-operative photographs of a patient with atypical SGC. ( $a$ and b) Upper lid mass seen in the peri punctalregion with focal loss of eyelashes, (c) Histopathological photomicrograph showing the typical lobular pattern of SGC with central necrosis, (d) Histopathological photomicrograph section through the canaliculus showing normal structure and no invasion by the tumor cells, *: canalicular lumen, (e and f) Post-operative external photograph showing good cosmesis and contour of the eyelid after reconstruction

using skin from the same upper eyelid through a blepharoplasty incision. The sliding tarso-conjunctival flap was secured to the posterior lacrimal crest to reconstruct the sharp angle of the medial canthus. Histopathological examination showed tumor consisting of irregular lobules and sheets of cells with central area of necrosis and centrally located nuclei with foamy, multivacuolated cytoplasm, secondary to intracytoplasmic lipid [Figure 1c]. Additional sections through the canaliculus, showed no evidence of tumor cells in the lumen or the epithelium [Figure 1d]. The cut end of the canaliculus was free of tumor cells. At the 6 week, post-operative visit the patient complained of minimal tearing. The eyelid margin was intact with a good contour and there was no clinical evidence of recurrence [Figure 1e]. At the last follow up, 24 months post surgery, the patient was noted to be recurrence free clinically, with good eyelid cosmesis [Figure 1f].

Sebaceous gland carcinoma of the eyelid is more commonly seen in older individuals beyond the age of 55 years. ${ }^{[1,2]}$ It has a female preponderance and is also more common in the Indian subcontinent. ${ }^{[1]}$ It is often clinically misdiagnosed because it can masquerade as a recurrent chalazion. Histopathological misdiagnosis is also well known with certain studies showing the misdiagnosis rate ranging from 40 to $75 \% .{ }^{[2]}$ This could be attributed to the inexperience and inadequate exposure of the general pathologist to these lesions. Both clinical as well as histopathological misdiagnosis can be avoided by good clinical evaluation by an experienced ocular oncologist followed by examination of the sections by an experienced pathologist using special stains such as oil red $\mathrm{O}$ and adipophilin. ${ }^{[5]}$

Our patient was a young female with history of incision and curettage done twice before she presented to us. Thus SGC was kept as our first differential diagnosis. In view of the patient's apprehension regarding an extensive surgical procedure, an incision biopsy from the peri-punctal area under frozen section control was initially planned. As the frozen section revealed the tumor to be a SGC a total tumor excision with wide margins and eyelid reconstruction was performed in the same sitting. In view of the peri-punctal location the option of excising the lacrimal drainage system completely and performing a dacryocystectomy in the same sitting was initially thought of and discussed with the patient. A previous case report has shown SGC to metastasize to the lacrimal sac 5 years after removal of the tumor. ${ }^{[6]}$ It is postulated that tumor cells spread via contiguous epithelial spread or free-floating cancer cells are implanted into the lacrimal drainage system via the tear fluid. ${ }^{[2]}$ However the exact mechanism remains unclear. In patients with a history of bloody epiphora saline irrigation should be avoided. Also in cases with a peripunctal mass, saline irrigation should be avoided through the affected punctum. Considering the young age of the patient, and T2a stage of the tumor (AJCC $7^{\text {th }}$ edition) a dacryocystectomy in the primary sitting was avoided. ${ }^{[7]}$ Serial histopathological sections were taken through the canaliculus to look for skip lesions and exclude the spread along the epithelium.

Our patient did not undergo a map biopsy in the primary sitting and the authors acknowledge this short-coming. However, over a follow-up of 2 years, systemic metastatic workup with PET scan remained negative. Sentinal lymph node biopsy was not performed in accordance with prognostic value of the staging system for eyelid tumors in the 7th Edition of the American Joint Committee on Cancer Staging Manual. ${ }^{[8]}$ Future follow up would include examination of the eyelid and the lacrimal sac area for the presence of any mass lesion.

This case describes clinical diagnosis and management of a very rare peri-punctal presentation of SGC. High degree of suspicion, astute clinical skills, good pathology back up and use of basic plastic surgery principles is imperative in managing such lesions.

\section{Acknowledgement}

We acknowledge the help of Mr SBN Chary and Mr Gattu Naresh in processing the photographs.

\section{Financial support and sponsorship}

This Study is funded by the Hyderabad Eye Research Foundation.

\section{Conflicts of interest}

There are no conflicts of interest. 


\section{RefEREnCES}

1. Sihota R, Tandon K, Betharia SM, Arora R. Malignant eyelid tumors in an Indian population. Arch Ophthalmol 1996;114:108-9.

2. Shields JA, Demirci H, Marr BP, Eagle RC Jr, et al. Sebaceous carcinoma of the ocular region: A Review. Surv Ophthalmol 2005;50:103-122.

3. Kaliki S, Ayyar A, Dave TV, Ali MJ, Mishra DK, Naik MN. Sebaceous gland carcinoma of the eyelid: Clinicopathological features and outcome in Asian Indians. Eye 2015;29:958-63.

4. Shields JA, Demirci H, Marr BP, et al. Sebaceous carcinoma of the eyelids: Personal experience with 60 cases. Ophthalmology 2004;111:2151-7.

5. Jakobiec FA, Mendoza PR. Eyelid sebaceous carcinoma:
Clinicopathologic and multiparametric immunohistochemical analysis that includes adipophilin. Am J Ophthalmol 2014;157:186-208.

6. Rath S, Honavar S, Reddy V. Sebaceous carcinoma of the eyelid metastasizing to the larimal sac after 5 years. Orbit 2009;28:309-12

7. Kaliki S, Gupta A, Ali MH, Ayyar A, Naik MN. Prognosis of eyelid sebaceous gland carcinoma based on the tumor (T) category of the American Joint Committee on Cancer (AJCC) classification. Int Ophthalmol. 2016;36:681-90.

8. Ford J, Thakar S, Thuro B, Esmaeli B. Prognostic Value of the Staging System for Eyelid Tumors in the 7th Edition of the American Joint Committee on Cancer Staging Manual. Ophthal Plast Reconstr Surg. 2017;33:317-324 